\title{
Orales Propranolol wirkt bei Blutschwämmchen
}

Eine groß angelegte Studie weist die regressive Wirksamkeit von oral verab-
reichtem Propranolol bei Hämangiomen im Säuglingsalter nach. Der Beta-
blocker wird in einer Dosierung von $3 \mathrm{mg} / \mathrm{kg} \mathrm{KG}$ über sechs Monate gegeben.

— Hämangiome sind die häufigsten Weichteiltumoren im Säuglingsalter. Meist treten sie erst zwischen der 4. und 6. Lebenswoche auf. Nach einer Proliferationsphase kommt es meist zur Stabilisation und zur Involution. Etwa 12\% der Kinder zeigen jedoch Komplikationen, einige auch Exazerbationen.

Glukokortikoide und bei deren Versagen Interferon alfa sowie Vincristin waren trotz ihres relativ großen Nebenwirkungspotenzials lange Mittel der Wahl, ohne dass ihre Effizienz belegbar gewesen wäre. Seit 2008 liegen die Hoffnungen auf dem systemisch verabreichten, nicht-selektiven Betablocker Propranolol. Eine weltweit durchgeführte, multizentrische, placebokontrollierte, randomisierte Studie an 460 Säuglingen untersuchte nun die Wirkung von oral verabreichtem Propranolol.

Säuglinge im Alter von 1-5 Monaten mit einem proliferierenden Hämangiom im Gesicht (70\%) oder an anderen Körperstellen $(30 \%)$ erhielten oral über einen Zeitraum von drei oder sechs Monaten entweder Placebo oder Propranolol in einer Dosierung von täglich 1 oder $3 \mathrm{mg} / \mathrm{kg}$ KG. Als Endpunkt der Studie galt eine komplette oder fast vollständige Remission der Hämangiome.

In der Propranolol-Gruppe konnte unter der Dosis von $3 \mathrm{mg} / \mathrm{kg} \mathrm{KG}$ ein signifikant größerer Effekt als in der Placebogruppe beobachtet werden (60 vs. $4 \%, \mathrm{p}<0,001) .88 \%$ dieser Patienten gegenüber $5 \%$ in der Placebogruppe hatten bereits nach einer dreimonatigen Therapie unter dieser Dosis eine Teilremission gezeigt. Insgesamt konnte aber nur bei 3 von 39 Patienten (8\%) nach drei Monaten der Endpunkt erreicht werden - bei der sechsmonatigen Therapie waren es 27 von 43 Patienten (63\%). 10\% der Patienten, die sich einer primär erfolgreichen Therapie unterzogen hatten, benötigten eine erneute systemische Therapie

in der Follow-up-Periode. Die für Propranalol beschriebenen Nebenwirkungen Hypoglykämie, Hypotonie, Bradykardie oder Bronchospasmen kamen unter Verum und Placebo gleich oft vor.

\section{- Léauté-Labrèze Cet al. \\ A Randomized, Controlled Trial of Oral Propranolol in Infantile Hemangioma. N Engl J Med. 2015;372:735-46}

\section{Kommentar}

Die Studie an einem großen Kollektiv von Säuglingen mit komplizierten Hämangio-

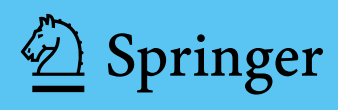

men belegt die Wirksamkeit einer oralen Therapie von $3 \mathrm{mg} / \mathrm{kg}$ KG über sechs Monate. Niedrigere Dosierungen oder kürzere Therapiedauer waren nicht effektiv. Leider wurde eine Dosis von $2 \mathrm{mg}$, die möglichweise schon wirksam gewesen wäre, nicht getestet. Auch konnten Hochrisikopatienten aufgrund der Placebo-Anlage der Studie nicht einbezogen werden. Die Autoren verweisen aber auf Einzelfallstudien, die auch z. B. bei Ulzerationen oder Lokalisation in Augennähe Erfolge nachweisen konnten. Die 47 Autoren empfehlen die Therapie bei komplizierten bzw. progredienten Hämangiomen im Säuglingsalter - unter engmaschinger Kontrolle.

Prof. Dr. med. Dr. h. c. D. Reinhardt .

\section{Hier steht eine Anzeige.}

